\title{
Hypersonic Free Flight Investigation on Rudder Reflection of Aircraft ${ }^{+}$
}

\author{
Fei Xue *, Yuchao Wang, Zenghui Jiang and Yinong Yang \\ China Academy of Aerospace Aerodynamics, Beijing 100074, China; zjf8763871@163.com (Y.W.); \\ 15201661429@163.com (Z.J.); xue_fei_88@163.com (Y.Y.) \\ * Correspondence: 12.9258@163.com; Tel.: +86-152-0166-1429 \\ + Presented at the 18th International Conference on Experimental Mechanics (ICEM18), Brussels, Belgium, \\ 1-5 July 2018.
}

Published: 12 July 2018

\begin{abstract}
In order to study the control effect of the rudder surface of the hypersonic vehicle and the coupling dynamic characteristics of the rudder surface deflection and the flight attitude, a technical platform for the deflection and motion coupling of the aircraft rudder surface was designed. The platform ejection mechanism can launch the model into the wind tunnel flow field according to the preset attitude, and model can free flight without support interference. The innovative design of the model internal rudder partial system can guarantee the model to deflect the rudder surface in the free flight process, simulate the real steering process of the aircraft. By changing spring with different springs, the speed of the rudder surface can be changed. The dual optical path and image acquisition technology can capture the motion picture before and after the deflection of the rudder surface from two angles. After the image is matched by model matching, the six degrees of freedom parameter of the model can be changed with the time curve before and after the deflection of the rudder surface, and the area of the six freedom degree curve of the different state model is compared. In other words, the specific influence of dynamic rudder rotation on the motion of the model is known. The wind tunnel test of the model in the hypersonic wind tunnel of the $500 \mathrm{~mm}$ is carried out using this platform. The test results are highly repeatable, and the test platform technology is mature and reliable.
\end{abstract}

Keywords: wind tunnel test; free flight; rudder reflection; hypersonic; unsteady aerodynamic

\section{Introduction}

The rudder surface is the main control surface of the aircraft. In order to improve mobility, most of the aircraft rely on the deflection of the rudder surface to provide a variety of control torque. However, with the increase of Mach number, the aerodynamic efficiency of the rudder will obviously decrease, which seriously affects the control characteristics of the rudder, and directly threatens the flight stability and maneuverability of the aircraft [1,2].

A variety of existing hypersonic vehicles still use the rudder surface to provide control torque [3]. In view of the efficiency of the rudder surface of a hypersonic vehicle, the main research means of the research institutions are the constant technique of hinges [4,5]. Although the high Mach number test can be carried out, the dynamic coupling dynamic problem of the control and motion after the deflection of the rudder surface is not considered. In recent years, the wind tunnel virtual flight test technology has successfully solved the above shortcomings, expanding the traditional wind tunnel test to the integrated level of flow, flight and control [6-10]. But the wind tunnel virtual flight test technology is only capable of subsonic speed [11-13]. It is well known that the hypersonic velocity is distinct from the aerodynamic characteristics of low velocity and subsonic speed, which makes the control problem of the aircraft rudder difference. Above all, we can see that the research 
ability of hinge force measurement and wind tunnel virtual flight test technology is limited for the control effect of supersonic and hypersonic vehicle's rudder control, while the control effect of rudder surface is the most likely problem when hypersonic. Therefore, it is urgent to study the dynamic control effect of the rudder surface of hypersonic vehicle, which combines the deflection of the rudder surface with the coupling motion of the aircraft.

Free flight test in wind tunnel is a common test method on unsteady aerodynamic phenomenon [14-16]. The China Academy of Aerospace Aerodynamics has accumulated rich experience in the field of free flight in supersonic and hypersonic wind tunnels. However, the conventional wind tunnel free flight test technology is not suitable for the study of the problem of deflection and motion coupling of the rudder surface, and the previous test technology should be improved. This paper will focus on research, a hypersonic condition on aircraft rudder deflection control coupling effect and motion unsteady test technology, and test research for rudder surface deflection of a plane symmetric hypersonic aircraft model in wind tunnel free flight process.

\section{Wind Tunnel for Test}

The test was carried out in the FD-07 wind tunnel of The China Academy of Aerospace Aerodynamics. The test parameters are shown in Table 1. The Maher number was changed by replacing the nozzle in the wind tunnel. The Ma range 4.5 to 10, the outlet diameter of the nozzle was $500 \mathrm{~mm}$, the free jet test section with the closed chamber was $1880 \mathrm{~m} \times 1400 \mathrm{~mm} \times 1130 \mathrm{~mm}$. At present, the angle of attack of the insertion mechanism is -10 degrees 50 degrees, the variation range of the sideslip angle is -10 degree 10 degrees, and can be moved before and after. Because the angle of the head angle of the angle of attack mechanism is large, the wind tunnel generally defines the angle of attack as positive when the head is lowered.

Table 1. FD-07 wind tunnel parameters.

\begin{tabular}{cccccc}
\hline Ma & P0/MPa & q $\infty / \mathbf{M P a}$ & $\mathbf{P} \infty / \mathbf{P a}$ & $\mathbf{R e} / \mathbf{1} / \mathbf{m}$ & $\mathrm{T}^{\mathbf{0}} /{ }^{\circ} \mathbf{C}$ \\
\hline 7.995 & 8.0 & 0.037 & 820 & $1.77 \times 10^{7}$ & 483 \\
\hline
\end{tabular}

\section{Wind Tunnel Test System}

The test system mainly consists of 3 parts: the model release system, the deflection system in the model, the optical path and the image acquisition system. The main task of the model release system is to launch the model into the wind tunnel flow field, and the internal rudder rotation system of the free flight model ensures the model's deflection on the rudder surface in full free state, and the optical path and image acquisition system can ensure that the experiment can obtain a clear model motion picture and analyze the motion trajectory and posture of the model from the model. The angle of state.

\subsection{Model Release System}

The main difficulty in the design improvement of the model emission system is that the model should be launched into the flow field in a very short time in accordance with the predetermined speed (5 15 m/s) and the angle of attack (-10 degree 10 degree), and the launching speed and angle of attack need to have good repeatability, so the emitter needs to have good repeatability and reliable operating stability. The rudder surface should be deflected in the free condition, and the model is supported by the launching mechanism before free flight, so the steering gear deflection trigger is best attached to the launching mechanism, which also increases the difficulty of the design of the launching mechanism.

When the test is carried out, the piston propulsion model moves in the direction of the reverse air flow, and the launching paws will automatically open when model moves forward. The model does not support the free movement in the flow field. And completes the launch and release of the model. In view of the characteristics of this test, the pullout line is added to the test device, as shown in Figure 1. When the model is free to fly to the limit of the length of the pull out line, the model is 
pulled out and the rudder rotation system is triggered, and the rudder deflection of the model is completed. The axis of the pin is over the model center of mass, so that the maximum effect on the motion posture of the model is smaller. In the experiment, two high-speed cameras were used to record the model attitude.

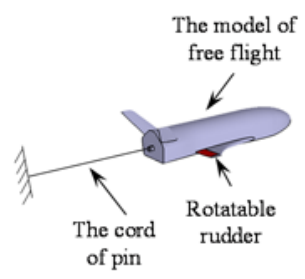

Figure 1. The trigger of rudder reflection.

\subsection{The Deflection System in the Model}

The free flight model rudder deflection system is the biggest difficulty of this test. First, the model needs to be deflected on the rudder surface in the complete free state of the launching mechanism, which puts forward a big problem for the design of the trigger mechanism. Secondly, the free flight model size is limited by the effective flow field and the performance of the launch system, and the model size is generally small. In this test, the free flight of the FD-07 wind tunnel is carried out in this test. The maximum size of the model is $100 \mathrm{~mm} \times 80 \mathrm{~mm} \times 40 \mathrm{~mm}$. Due to the thinner wing and head of the aircraft, the space that can be used to install the actuator is far less than the above size, and the difficulty of the model design is increased. Again, the wind tunnel free flight test is different from the static force test, and the latter can be installed on the outside of the model and free flying model. In addition, the free flight model needs to have similar quality distribution characteristics to the actual model, including the model center of mass and the value of inertia, so the model quality should be considered at the same time in the design of the internal mechanism of the model. The requirement of parameters increases the difficulty of model design. Finally, the free flying rudder deviation control technology of the model requires that the deflection speed of 2 rudder surfaces and the angle of 2 rudder surfaces are simulated, and the rudder deflection is guaranteed after the deflection of the rudder surface. The mechanism needs to resist the wind tunnel air flow ability, prevent the rudder back deviation, and increase the difficulty of the model design.

In view of the above 5 main difficulties, a set of free flight model rudder deflection system is designed and innovating in this experiment. The whole body is controlled by the pullout. The compression spring will drive the rotating shaft block and the rudder shaft together. The two rudder surfaces are connected by the same shaft block to ensure the real-time synchronism of the rotation of the two rudder surfaces, thus avoiding the aerodynamic interference caused by the deviation of the left and right rudders.

The deflection angle of the steering wheel and the rudder surface can be controlled by the steering angle control surface. The deflection angle of the rudder surface can be adjusted by adjusting the distance between the steering wheel and the axis of the pin hole. The effect of pulling off pin action on the free movement of the model can be reduced to a maximum by the axis of the pin hole. In the design of the model, the position of the rudder shaft of the model is determined, while the center of mass of the model determines the axis position of the pin. Using the three-dimensional model design software, the rudder surface is deflected to the final deflection position. At this time, the distance between the control surface of the rudder and the axis of the pin hole is adjusted so that the control surface is just in contact with the shaft block, and the distance between the steering axis of the rudder and the axis of the pin hole is determined. The purpose of simulating different rudder deflection speed or rudder deflection time can be achieved by using different spring springs. After the rudder deflection, the spring still has partial thrust to prevent the rudder from being deflected under aerodynamic force. The test shows that the full deployment time of pulling pin to rudder is about $0.001 \mathrm{~s}$. Through this system, the rudder deflection can be realized in the free flight of the model. 


\subsection{Optical System and Image Acquisition System}

In order to better identify the six degree of freedom parameter of the model free flight process, this experiment carried out a dual angle optical path design for FD-07 wind tunnel. The optical path design needs to overcome the limited space in the wind tunnel, and install the light source and reflector in the wind tunnel. It not only needs to ensure the normal work of the equipment, but also can not produce interference in the convective field. In order to ensure the visibility of the view of the light path, the wind tunnel portal is redesigned and redesigned. On the premise of ensuring the safety of the test, a circular observation window of the original portal is adjusted to two square observation windows, which are used for the side optic road and the elevation light path respectively. Through this adjustment, we can ensure that two light paths are photographed at the same time, so as to capture the posture change characteristics of the model motion.

The dual angle optical path and acquisition system are mainly divided into two parts: side looking optical path and side looking camera, overlooking optical path and overlooking camera. As shown in Figure 2. The side view optical path uses the schlieren system of the wind tunnel itself, including its light source system, and the side view camera is used to record the side view image of the model. The position of the overlooking light source is shown in the diagram, and the light source is installed on the wind tunnel wall panel. The overlooking optical path refracts out of the hole through the reflector installed above the wind tunnel test section, and overlooks the image of the model through a vertical camera.

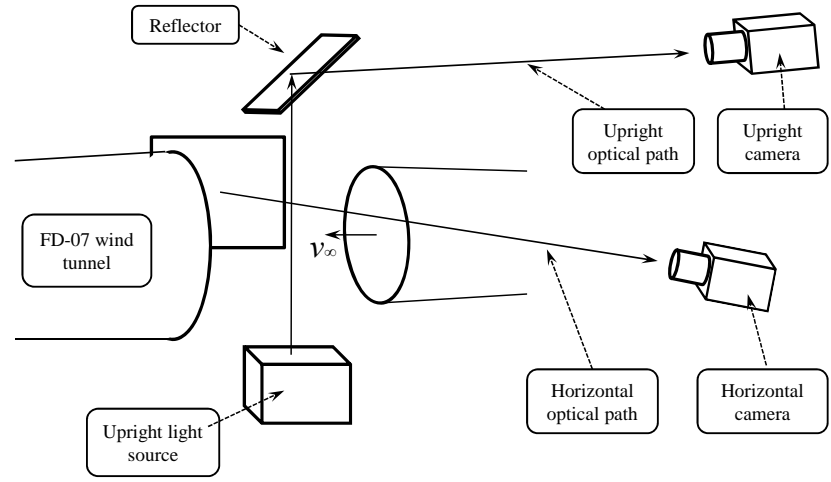

Figure 2. Upright and horizontal optical path system.

Attitude calibration can identify the 6 degree of freedom parameters of the missile after obtaining the picture. The calibration is to put a known 6 degree of freedom parameter model in the landing area of the missile, shoot the image by two cameras, and then match the image with the 6 degree of freedom component, thus the corresponding relationship between the image and the model 6 degree of freedom parameters is obtained.

The desired image is obtained through background subtraction, median filtering, shadow removal, mathematical morphology processing and connected domain analysis. The $X, Y, Z$ coordinates, pitch angle and yaw angle of the model centroid are identified by positive and inverted views, and then the model roll angle is obtained by model matching, and the six degree of freedom parameter of model motion is obtained.

\section{Reliability Test}

In order to test the reliability of the platform, a verification test was carried out. The $\boldsymbol{\alpha}_{0}$ is the initial angle of attack for the model, and the rising of the model is positive. $\theta$ is the deflection angle of the rudder and the rudder surface is positive. The time reference value $t_{0}=0.025 \mathrm{~s}$, the length reference value is the model length $l_{0}=100 \mathrm{~mm}$, the angle reference value takes the rudder deflection angle maximum value $\theta_{0}=15$ degree. The ejection velocity of the model is $\mathrm{v}$, which is about $8 \mathrm{~m} / \mathrm{s}$. The initial launch attack angle of the test model is 0 degrees, the deflection angle of the rudder surface is 15 degrees, the experiment is repeated once, the number is test 1 and test 2 respectively, 
and the test 3 is the fixed rudder partial state of the steering angle of 0 degrees, and the other parameters are the same as the previous two tests. The parameters of the test flow field are shown in Table 1 . The state description is shown in Table 2.

Table 2. Test conditions.

\begin{tabular}{ccccc}
\hline No. & $\mathbf{M a}$ & $\boldsymbol{\alpha}_{0} /$ Degree & $\boldsymbol{\theta} /$ Degree & Rudder \\
\hline 1 & 7.995 & 0 & 15 & Fixed \\
2 & 7.995 & 0 & $0 \sim 15$ & Adjustable \\
3 & 7.995 & 0 & 0 & Fixed \\
\hline
\end{tabular}

The coordinate system is shown in Figure 3. In order to make the experimental data comparable, the origin of the coordinate system is located on the central axis of the wind tunnel and $0.55^{*} l_{0}$ from the left side of the observation window. The $X$ axis is the original point of the coordinate system, the direction of the reverse flow is positive; the $Y$ axis over coordinate origin is vertical upward; the $Z$ axis is over the coordinate origin, and the positive direction of the $Z$ axis is determined according to the right rule; the direction of the pitching, yaw, and roll correction is determined according to the right rule. The pulling pin time is zero time, that is, $t=0$. Figure 4 is the curves of the model centroid position changing with time. The motion trajectories of test 1 and test 2 model coincide better, and the divergence of test 3 model with vertical rudder surface appears in the vertical direction.

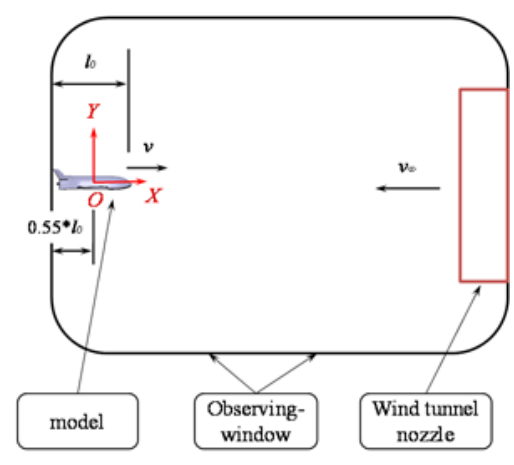

Figure 3. The coordinate system.

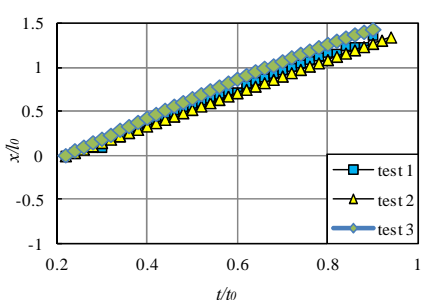

(a)

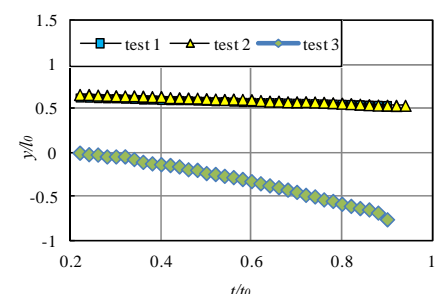

(b)

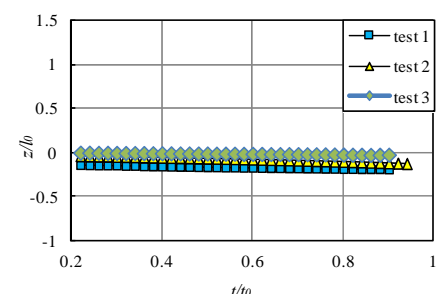

(c)

Figure 4. Curves of centroid position changing with time. (a) Curve of $X$ direction displacement changes with time; (b) Curve of $Y$ direction displacement changes with time; (c) Curve of $Z$ direction displacement changes with time.

Figure 5 is the curve of the model attitude angle with time. The change trend of the pitch curve and yaw angle curve of test 1 and test 2 model is the same. The test 3 rudder surface is not deflected, the model is much lower and the angle of attack diverges. At the same time, the angle of attack divergence causes the $X$ and $Y$ movement to increase. Figure $5 \mathrm{c}$ is the roll angle change curve of the 3 test. From the graph, it can be seen that the roll angle curves of the first two tests are different and the curves are less smooth. This is due to the inertia of the model around the 3 axis, the inertia of the rotation axis is the least, usually one order of magnitude lower than the other 2 axis inertia, and the smaller rolling torque will bring a larger roll angle. Moreover, although the optical path system has 
been greatly improved, the angle of view has increased to 2, which are side looking and overlooking. However, in these 2 angles, there is a shortage of the roll angle of the recognition model. The best way to improve the accuracy of the roll angle recognition is to add the visual angle of view, and the direction of the face is exactly the direction of the air flow, and it is more difficult to add the visual angle.

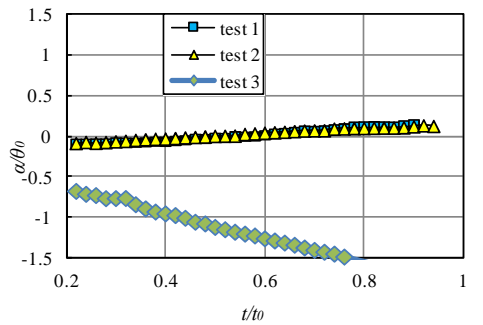

(a)

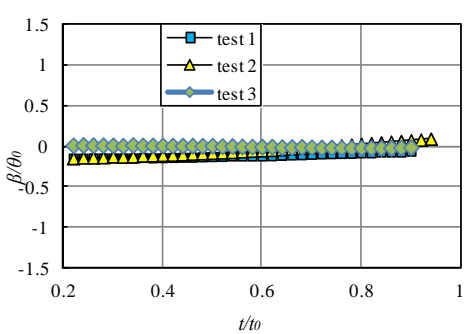

(b)

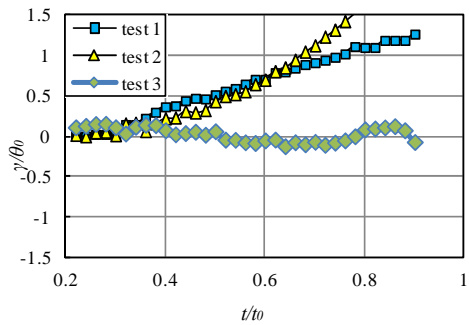

(c)

Figure 5. Curves of angle changing with time. (a) Angle of attack with time change curve; (b) The curve of the yaw angle with time; (c) Roll angle change curve with time.

The existing test technology of the platform can get six degrees of freedom parameters of different state models, and the influence of rudder rotation parameters on motion can be obtained through comparison. However, the existing analysis is only qualitative analysis, and the analysis is inaccurate, not quantitative. The next step is to identify the parameters of the test data. By identifying the six degree of freedom parameter curve of the model, the aerodynamic force and torque variation of the model are obtained, and the influence of a variety of dynamic rudder rotation on the aerodynamic force of the model is further obtained.

\section{Conclusions}

In view of the control effect of the rudder surface of the hypersonic vehicle and the coupling characteristics of the rudder rotation and the flight attitude, a technical platform for the deflection and motion coupling of the aircraft rudder surface is established. The model rudder rotation system of innovative design enables the model to deflect the rudder surface in the free flight and simulate the real rudder process of the aircraft, and the dual optical path and image acquisition technology can obtain the six degrees of freedom parameters of the model with the time change curve before and after the deflection of the rudder surface, and compare the difference of the six degrees of freedom curve of different state models and know the dynamics. The effect of the rudder rotation on the motion of the model. The test shows that the platform technology is mature and reliable, which meets the purpose of testing the control effect of the dynamic rudder of the aircraft under the hypersonic condition, and has made remarkable progress in the wind tunnel test technology in this field.

\section{References}

1. Xiong, L.; Liu, Z.; Chen, H.W. Hinge moment balance technique and application in hypersonic wind tunnel. Exp. Fluid Mech. 2007, 21, 54-57.

2. Chen, H.W.; Liu, Z.; Xiong, L. Double balance technology and its application on control surface force test in hypersonic wind tunnel. Exp. Fluid Mech. 2011, 25, 76-78.

3. Li, Q.; Shi, J. Recent developments of hypersonic technology. Struct. Environ. Eng. 2012, 39, 55-64.

4. Liu, X.H.; Wang, T.H.; Qiu, J.W.; Li, S.W.; Feng, X.H. Hinge moment balance technique with axial force measurement and its application in wind tunnel test. Exp. Fluid Mech. 2011, 25, 88-91.

5. Chen, J.Z.; Zhao, Z.I.; Tu, Z.G.; Jiang, M.H.; Yi, G.Q.; Yang, H.Y. Test technique investigation for aerodynamics and controlling capability of a grid fin in speed wind tunnel. Exp. Fluid Mech. 2011, 25, $82-87$.

6. Owens, B.; Brandon, J.; Croom, M.; Fremaux, M.; Heim, G.; Vicroy, D. Overview of Dynamic Test Techniques for Flight Dynamics Research at NASA LaRC. In Proceedings of the 25th AIAA Aerodynamic Measurement Technology and Ground Testing Conference, San Francisco, CA, USA, 5-8 June 2006; AIAA2006-3146; ARC: Reston, VA, USA, 2006. 
7. Smith, C.C. Flight Tests of a 16 -Scale Model of the Hawker P 1127 Jet VTOL Airplane; NASA TM SX-531; NASA: Washington, DC, USA, 1961.

8. Mullin, S.N. The Evolution of the F-22 Advanced Tactical Fighter; AIAA92-4188; Wright Brothers Lecture: Washington, DC, USA, 1992.

9. Jackson, E.B.; Buttrill, C.W. Control Laws for a Wind Tunnel Free-Flight Study of a Blended-Wing-Body Aircraft; NASA/TM-2006-214501; NASA: Washington, DC, USA, 2006.

10. Yang, Y.; Wu, Z.; Yang, C. Control Surface Efficiency Analysis and Utilization of an Elastic Airplane for Maneuver Loads Alleviation; AIAA 2013-1487; ARC: Reston, VA, USA, 2013.

11. Sun, H.S.; Cen, F.; Nie, B.W.; Liu, Z.T. Present research status and prospective application of wind tunnel free flight test technique. Exp. Fluid Mech. 2011, 25, 103-108.

12. $\mathrm{Hu}, \mathrm{J} . ; \mathrm{Li}, \mathrm{Q}$. Primary investigation of the virtual $\mathrm{f}$ light testing techniques in wind tunnel. Exp. Fluid Mech. 2010, 24, 95-99.

13. Li, H.; Zhao, Z.L.; Fan, Z.L. Simulation method for wind tunnel based virtual flight testing. Exp. Fluid Mech. 2011, 25, 72-76.

14. Xue, F.; Jin, X.; Wang. Y. Experimental investigation on the high speed weapon delivery from inter-nal weapons bay. Acta Aeronaut. Astronaut. Sin. 2017, 38, 114-120.

15. Jiang, Z.H.; Chen, N. Wind tunnel free-flight test with biplanar optical system on the spinning blunt cone. Chin. Theor. Appl. Mech. 2013, 45, 777-781. (In Chinese)

16. Quyao, J.; Yinong, Y.; Zenghui, J. The Precision and Accuracy of Wind Tunnel Free-flight Experiment Result. Astronautics 2009, 30, 2082-2085.

(C) 2018 by the authors. Licensee MDPI, Basel, Switzerland. This article is an open access article distributed under the terms and conditions of the Creative Commons Attribution (CC BY) license (http://creativecommons.org/licenses/by/4.0/). 